\title{
The distribution of novae in the Magellanic Clouds
}

\author{
Raquel Yumi Shida \\ Departamento de Astronomia, IAG/USP, Rua do Matão 1226, Cidade \\ Universitária, 05508-900 São Paulo SP, Brazil \\ William Liller \\ Center for Nova Studies, Casilla 5022 Reñaca, Viña del Mar, Chile
}

\begin{abstract}
Some 34 novae have been discovered in the LMC and 14 in the SMC. Historically, the novae seemed to avoid the central bar of the LMC and the main body of the SMC, but with the novae discovered in the past two decades a different picture emerges. The more dramatic situation exists in the SMC where all seven of the most recent novae have occurred in or very near the main body. Indications are that the age of the LMC bar is around 5 Gyr (Smecker-Hane et al. 2002), certainly old enough to breed novae. Perhaps the puzzle now is why there have been so many novae in the disk component of the LMC where many H II regions are found.
\end{abstract}

\section{General picture: the LMC}

It has long been a puzzle as to why the central bar of the LMC almost seemed to be a "zone of avoidance" for novae. Recently, Shara (private communication) and Della Valle (private communication) have raised questions about possible observational effects and have suggested that novae in the central regions were simply being missed. Fig. 1 shows the locations of all 34 novae known or presumed to have occurred in the LMC (detailed data for these novae and for those in the SMC are in Liller \& Shida, these proceedings). One measure of the concentration to the bar is given by the standard deviations of the average positions of the novae. For the novae discovered prior to 1985, the average R.A. and Dec. are $5 \mathrm{~h} 23.8 \mathrm{~m} \pm 17.9 \mathrm{~m}$ and $-68.90^{\circ} \pm 1.89^{\circ}$, while for novae found afterwards these figures become $5 \mathrm{~h} 21.5 \mathrm{~m} \pm 10.5 \mathrm{~m}$ and $-70.22^{\circ} \pm 0.98^{\circ}$. (The 1935 nova at R.A. $3 \mathrm{~h} 59 \mathrm{~m}$ has been omitted because of its questionable association).

Clearly, the more recent novae have been found closer to the central bar, the change being caused, perhaps, by earlier photographic discoveries having been made on fully exposed plates which frequently burned out the central regions because their use was often for the study of faint variables. Also, some of the novae, especially those found by Graham (Graham \& Araya 1971), were detected spectroscopically, and the confusion in the central regions could very well have obscured faint new emission-line objects. Since 1985 nova hunters (Wischnjewsky, McNaught, Garradd, Liller) have used direct photographs ex- 


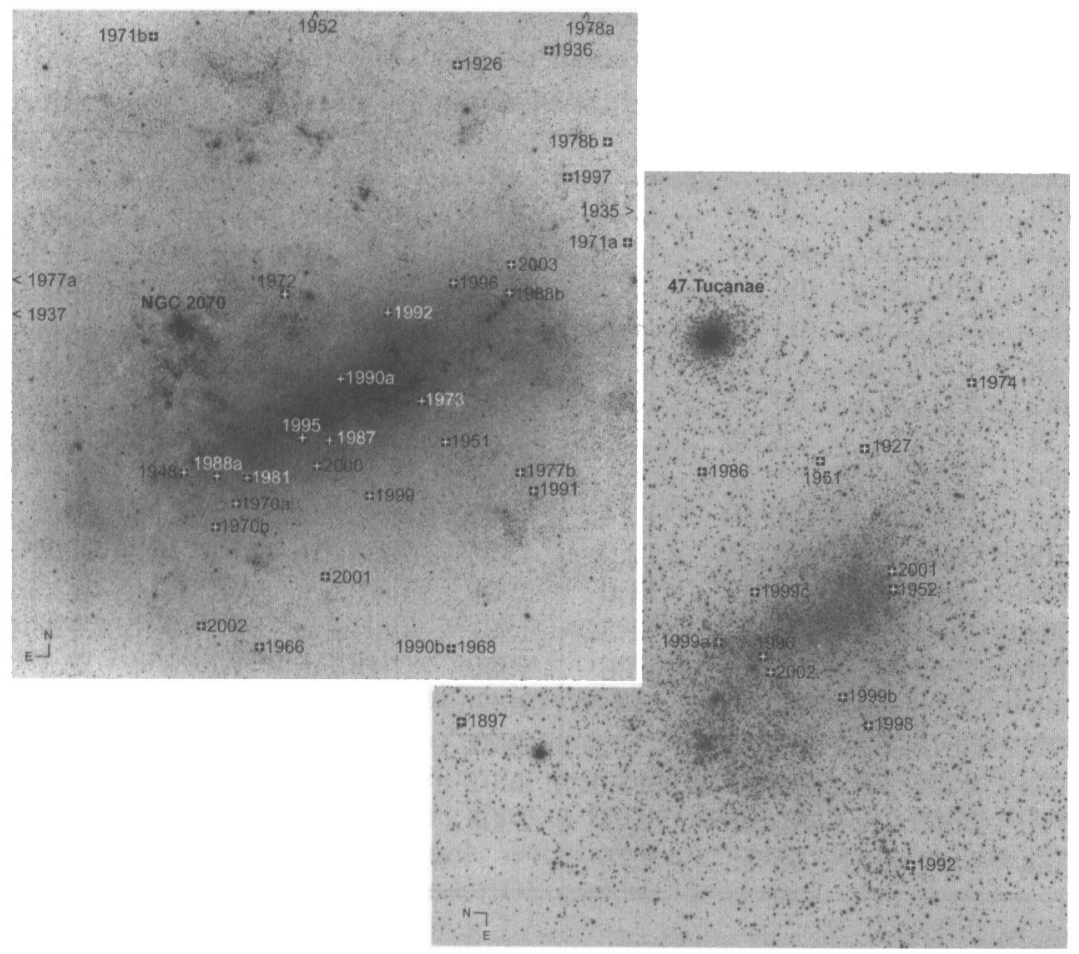

Figure 1. Novae in the LMC (left) and the SMC (right). The coordinates are given in Tables 1 and 2 .

posed to optimize search conditions in the bar and elsewhere. Additionally, the MACHO gravitational lens survey added one other faint nova. Also noteworthy is the shift of the average positions of older and more recent novae amounting to $67^{\prime}$ to the southwest. Why this should be is difficult to understand, but it may be because of greater interest of earlier investigators in the richer regions north of the central bar where the remnants of a spiral arm can be seen.

In our Galaxy, classical novae can be classified as "intermediate Population II" with locations in the thick disk and with lesser amounts of hydrogen $\left(\mathrm{X} / \mathrm{X}_{\odot}=\right.$ 0.62 according to Sparks et al. (1999a)). Indeed, these novae are found only moderately close to the galactic equator with $\langle|\mathrm{b}|\rangle=9.1^{\circ}$ (Sparks et al. 1999b). Thus, it would seem that the distribution of novae in the LMC is now closer to what would be expected.

\section{General properties: the SMC}

A more dramatic situation exists in the SMC (see Fig. 1) where five (or six) of the nine most recently discovered novae lie in or very near the central body of this irregular galaxy. Remarkably, four of these were found in one year (1999) by gravitational lens surveys, two by MACHO and two by EROS2 (one of them had 
Table 1. Coordinates of known novae in the LMC (equinox 2000).

$V$ is the deduced maximum $V$ magnitude.

\begin{tabular}{cccc|cccc}
\hline YR & R.A. & DEC. & V & YR & R.A. & DEC. & V \\
\hline 1926 & $5 \mathrm{~h} 14.9 \mathrm{~m}$ & $-66^{\circ} 49^{\prime}$ & 12.0 & $1978 \mathrm{~b}$ & $5 \mathrm{~h} 01.5 \mathrm{~m}$ & $-67^{\circ} 19^{\prime}$ & $?$ \\
1935 & $3 \mathrm{~h} 59.6 \mathrm{~m}$ & $-67^{\circ} 47^{\prime}$ & 11.0 & 1981 & $5 \mathrm{~h} 32.1 \mathrm{~m}$ & $-70^{\circ} 22^{\prime}$ & $<12.0:$ \\
1936 & $5 \mathrm{~h} 07.4 \mathrm{~m}$ & $-66^{\circ} 39^{\prime}$ & 10.5 & 1987 & $5 \mathrm{~h} 24.2 \mathrm{~m}$ & $-70^{\circ} 02^{\prime}$ & 9.6 \\
1937 & $5 \mathrm{~h} 57.0 \mathrm{~m}$ & $-68^{\circ} 55^{\prime}$ & 10.6 & $1988 \mathrm{a}$ & $5 \mathrm{~h} 35.2 \mathrm{~m}$ & $-70^{\circ} 22^{\prime}$ & 11.0 \\
1948 & $5 \mathrm{~h} 38.3 \mathrm{~m}$ & $-70^{\circ} 21^{\prime}$ & 13.0 & $1988 \mathrm{~b}$ & $5 \mathrm{~h} 08.2 \mathrm{~m}$ & $-68^{\circ} 40^{\prime}$ & 10.0 \\
1951 & $5 \mathrm{~h} 12.8 \mathrm{~m}$ & $-69^{\circ} 58^{\prime}$ & 11.9 & $1990 \mathrm{a}$ & $5 \mathrm{~h} 23.4 \mathrm{~m}$ & $-69^{\circ} 30^{\prime}$ & 9.7 \\
1952 & $5 \mathrm{~h} 27.9 \mathrm{~m}$ & $-66^{\circ} 05^{\prime}$ & $<11.4$ & $1990 \mathrm{~b}$ & $5 \mathrm{~h} 10.0 \mathrm{~m}$ & $-71^{\circ} 39^{\prime}$ & 10.2 \\
1966 & $5 \mathrm{~h} 30.5 \mathrm{~m}$ & $-71^{\circ} 46^{\prime}$ & $<11.1$ & 1991 & $5 \mathrm{~h} 03.7 \mathrm{~m}$ & $-70^{\circ} 18^{\prime}$ & 8.8 \\
1968 & $5 \mathrm{~h} 10.0 \mathrm{~m}$ & $-71^{\circ} 40^{\prime}$ & 10.4 & 1992 & $5 \mathrm{~h} 19.4 \mathrm{~m}$ & $-68^{\circ} 55^{\prime}$ & 10.2 \\
$1970 \mathrm{a}$ & $5 \mathrm{~h} 33.2 \mathrm{~m}$ & $-70^{\circ} 35^{\prime}$ & 12.0 & 1995 & $5 \mathrm{~h} 26.8 \mathrm{~m}$ & $-70^{\circ} 01^{\prime}$ & 10.2 \\
$1970 \mathrm{~b}$ & $5 \mathrm{~h} 35.2 \mathrm{~m}$ & $-70^{\circ} 47^{\prime}$ & 11.0 & 1996 & $5 \mathrm{~h} 13.5 \mathrm{~m}$ & $-68^{\circ} 38^{\prime}$ & $<12.4$ \\
$1971 \mathrm{a}$ & $4 \mathrm{~h} 58.3 \mathrm{~m}$ & $-68^{\circ} 06^{\prime}$ & 11.8 & 1997 & $5 \mathrm{~h} 04.4 \mathrm{~m}$ & $-67^{\circ} 39^{\prime}$ & $<12.7$ \\
$1971 \mathrm{~b}$ & $5 \mathrm{~h} 40.6 \mathrm{~m}$ & $-66^{\circ} 40^{\prime}$ & 13.0 & 1999 & $5 \mathrm{~h} 19.9 \mathrm{~m}$ & $-70^{\circ} 28^{\prime}$ & 12.5 \\
1972 & $5 \mathrm{~h} 28.7 \mathrm{~m}$ & $-68^{\circ} 49^{\prime}$ & $<11.0$ & 2000 & $5 \mathrm{~h} 25.2 \mathrm{~m}$ & $-70^{\circ} 15^{\prime}$ & 10.1 \\
1973 & $5 \mathrm{~h} 15.3 \mathrm{~m}$ & $-69^{\circ} 39^{\prime}$ & $<10.9$ & 2001 & $5 \mathrm{~h} 24.0 \mathrm{~m}$ & $-71^{\circ} 10^{\prime}$ & $<9.7$ \\
$1977 \mathrm{a}$ & $6 \mathrm{~h} 05.8 \mathrm{~m}$ & $-68^{\circ} 38^{\prime}$ & $<13.0:$ & 2002 & $5 \mathrm{~h} 36.8 \mathrm{~m}$ & $-71^{\circ} 36^{\prime}$ & 10.1 \\
$1977 \mathrm{~b}$ & $5 \mathrm{~h} 05.2 \mathrm{~m}$ & $-70^{\circ} 09^{\prime}$ & 10.7 & 2003 & $5 \mathrm{~h} 08.4 \mathrm{~m}$ & $-68^{\circ} 26^{\prime}$ & $<11.0:$ \\
$1978 \mathrm{a}$ & $5 \mathrm{~h} 05.9 \mathrm{~m}$ & $-65^{\circ} 53^{\prime}$ & 9.75 & & & & \\
\hline \hline
\end{tabular}

its outburst in 1998). Only one of six novae found previously (1952) occurred near the central mass; the rest were well outside the boundary. Again, we believe the reason for this apparent dichotomy is that because earlier photographic plates were deeply exposed to be used for studies of faint variables, and as a result the central regions of the SMC were greatly overexposed and probably under-searched.

Table 2. Coordinates of known novae in the SMC (equinox 2000). $V$ is the deduced maximum $V$ magnitude

\begin{tabular}{cccc|cccc}
\hline YR & R.A. & DEC. & V & YR & R.A. & DEC. & V \\
\hline 1897 & $1 \mathrm{~h} 00.0 \mathrm{~m}$ & $-70^{\circ} 15^{\prime}$ & 11.0 & 1996 & $0 \mathrm{~h} 54.7 \mathrm{~m}$ & $-72^{\circ} 32^{\prime}$ & $<13.5$ \\
1927 & $0 \mathrm{~h} 34.2 \mathrm{~m}$ & $-73^{\circ} \circ 6^{\prime}$ & 11.4 & 1998 & $1 \mathrm{~h} 02.4 \mathrm{~m}$ & $-73^{\circ} 18^{\prime}$ & $<13.0$ \\
1951 & $0 \mathrm{~h} 35.3 \mathrm{~m}$ & $-72^{\circ} 58^{\prime}$ & 11.5 & $1999 \mathrm{a}$ & $0 \mathrm{~h} 53.7 \mathrm{~m}$ & $-72^{\circ} 13^{\prime}$ & 13.6 \\
1952 & $0 \mathrm{~h} 48.5 \mathrm{~m}$ & $-73^{\circ} 31^{\prime}$ & 11.0 & $1999 \mathrm{~b}$ & $0 \mathrm{~h} 59.4 \mathrm{~m}$ & $-73^{\circ} 08^{\prime}$ & $12.8:$ \\
1974 & $0 \mathrm{~h} 26.1 \mathrm{~m}$ & $-74^{\circ} 02^{\prime}$ & $<11.0:$ & $1999 \mathrm{c}$ & $0 \mathrm{~h} 48.9 \mathrm{~m}$ & $-72^{\circ} 30^{\prime}$ & $<13.0$ \\
1986 & $0 \mathrm{~h} 36.9 \mathrm{~m}$ & $-72^{\circ} 05^{\prime}$ & 10.1 & 2001 & $0 \mathrm{~h} 46.5 \mathrm{~m}$ & $-73^{\circ} 30^{\prime}$ & 11.8 \\
1992 & $1 \mathrm{~h} 17.7 \mathrm{~m}$ & $-73^{\circ} 31^{\prime}$ & $?$ & 2002 & $0 \mathrm{~h} 56.5 \mathrm{~m}$ & $-72^{\circ} 36^{\prime}$ & $11.8:$ \\
\hline \hline
\end{tabular}




\section{Discussion}

According to Smecker-Hane et al. (2002) indications are that the age of the LMC bar is around $5 \mathrm{Gyr}$, and Subramaniam \& Anupama (2002) concur, deducing that the bar is at least $4 \mathrm{Gyr}$ old. The latter authors further note that colormagnitude diagrams of stars near LMC novae suggest that star formation started between 4-2 Gyr ago leading one to conclude that novae should indeed be found in the bar. The question now would seem to be, why have so many novae been discovered in the younger disk component where many H II regions are found?

Systematic nova searches in the Magellanic Clouds continue (by Liller, at least), strong impetus having come from a keen desire to study new novae with the various space telescopes currently in operation.

\section{Acknowledgements}

This paper utilizes public domain data obtained by the MACHO Project, jointly funded by the US Department of Energy through the University of California, Lawrence Livermore National Laboratory under contract No. W-7405-Eng-48, by the National Science Foundation through the Center for Particle Astrophysics of the University of California under cooperative agreement AST-8809616, and by the Mount Stromlo and Siding Spring Observatory, part of the Australian National University. We also thank Wei-Hao Wang for providing photographs of the Magellanic Clouds and the EROS and MOA projects.

\section{References}

Graham, J.A., Araya, G. 1971, AJ, 76, 768

Smecker-Hane, T.A., Cole, A.A., Gallagher, J.S., Stetson, P.B. 2002, ApJ, 566, 239

Sparks, W.M., Starrfield, S.G., Sion, E.M., Shore, S.N., Chanmugam, G., Webbink, R.F. 1999a, in Allen's Astrophysical Quantities, 4th ed., ed. A.N. Cox, p. 436 (Springer-Verlag: New York)

Sparks, W.M., Starrfield, S.G., Sion, E.M., Shore, S.N., Chanmugam, G., Webbink, R.F. 1999b in Allen's Astrophysical Quantities, 4th ed., ed. A.N. Cox, p. 431. (Springer-Verlag: New York)

Subrarnaniam, A., Anupama, G.C. 2002, A\&A, 390, 449 


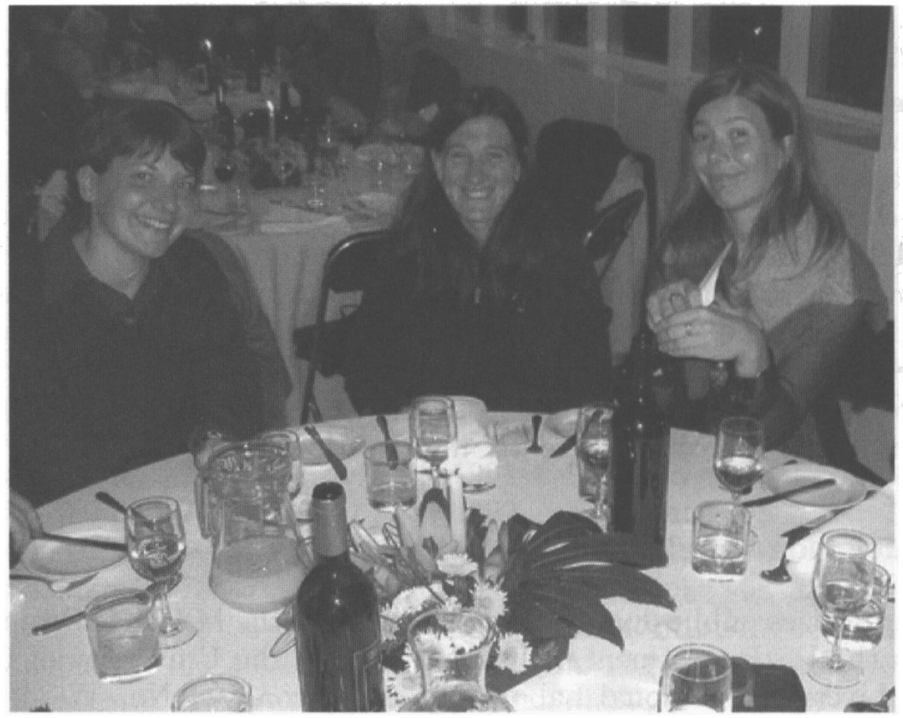

Marta Mottini, Lara Baldacci and Mari-Rosa Cioni

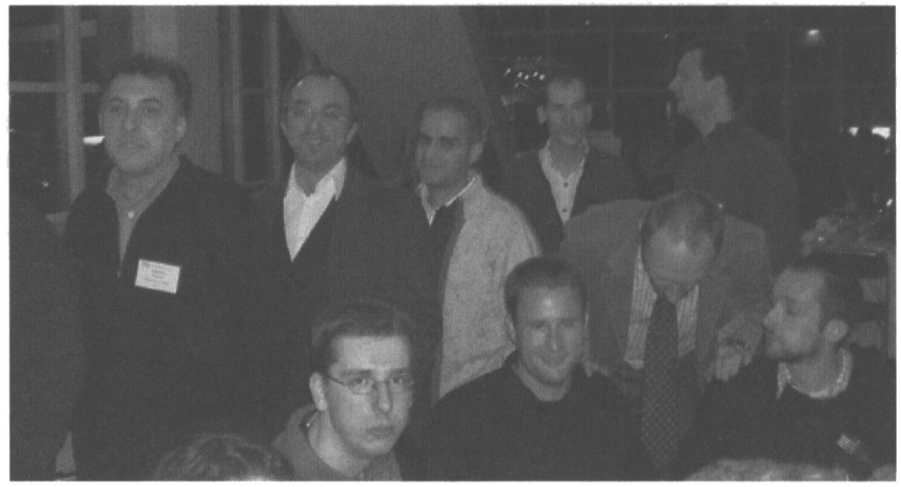

Antonio Aparicio, Guiseppe Bono, Joris De Ridder, Santi Cassisi, Torben Arentoft, Martin Groenewegen, Franz Kerschbaum, Martino Romaniello, Peter De Cat 